\title{
Denosumab-Related Osteonecrosis of the Jaw: a five year monocentric retrospective study of Ancona General Hospital
}

lucrezia togni ${ }^{1}$, Marco Mascitti, Andrea Tesei, Vittorio Zavaglia ${ }^{1}$, Alessandra Nori ${ }^{2}$, Andrea Santarelli ${ }^{1}$

1 Università Politecnica delle Marche

2 Azienda Ospedaliero Universitaria Ancona

Funding: The author(s) received no specific funding for this work.

Potential competing interests: The author(s) declared that no potential competing interests exist.

\section{Abstract}

Medication-Related Osteonecrosis of Jaws (MRONJ) represents a progressive destruction and death of bone affecting the jaws of patients treated by medications increasing the risk of disease, in the absence of a previous radiation therapy. Medications involved in the etiopathogenesis of MRONJ include antiresorptive drugs and biological agents. Its incidence and prevalence in general population are unknown and highly variable, due to the heterogeneity of available epidemiological data. MRONJ significantly affects the patient's quality of life (QoL), therefore it is necessary to improve the knowledge of its pathogenesis and lead the decision-making process of specialists. This retrospective study described the 5-years clinical experience of a monocentric Institute regarding Denosumab-related osteonecrosis of jaws cases. The study included all MRONJ cases of oncological and osteometabolic patients administered with Denosumab diagnosed from January 2014 to December 2018 in the Department of Special and Surgical Stomatology, "Ospedali Riuniti”, Ancona General Hospital. Our data showed a higher prevalence of MRONJ in female gender, in breast cancer patients and in subject administered with concurrent chemotherapy. Furthermore, the mandible represents the most affected site, pain was the most symptom complained, and stage 2 was the most common diagnosed. Despite the efficacy of prevention measures, the reduction of long-term bisphosphonates prescription, and the introduction of Denosumab, literature data showed a substantially unchanged number of new cases/year. Thus, it is fundamental to improve the scientific research, reporting any cases of new drugs, to clarify by evidence-based medicine all the controversial aspects of MRONJ.

Background: Medication-Related Osteonecrosis of Jaws (MRONJ) is an adverse drug reaction described as a progressive destruction and death of bone affecting the jaws of patients treated by medications increasing the risk of disease, in the absence of a previous radiation therapy. It is diagnosed and scored by clinic and radiographic examinations, regardless the presence of exposed necrotic bone or bone probing via sinus/fistula tracts for more than 8 weeks ${ }^{[1]}$. Medications involved in the etiopathogenesis of MRONJ include antiresorptive drugs, such as bisphosphonates (BFs) and Denosumab, and biological agents such as the inhibitors of angiogenesis, the tyrosine kinase inhibitors, and the inhibitors of mammalian target of rapamycin. The antiresorptive drugs are bone targeting agents used to prevent skeletonrelated adverse events and complications of oncological and metabolic disease ${ }^{[2]}$. BFs are analogues of inorganic 
pyrophosphate, inhibiting the pyrophosphate-dependent enzymes mediating bone resorption, whereas Denosumab is a monoclonal human IgG2 antibody binding the receptor activator of nuclear factor-kB ligand (RANK-L), inhibiting the osteoclasts recruitment and activation, and reducing the bone turnover. The MRONJ cases have been described in all categories patients treated by antiresorptive drugs and, with a lower incidence, by biological agents ${ }^{[3]}$. However, their real incidence and prevalence in general population are unknown and highly variable, due to the lack of available epidemiological data. Although notable progress has been made, many controversial issues regarding its pathogenesis, diagnosis, and treatment, still remained. A multifactorial pathogenesis has been suggested, involving the inhibition of angiogenesis, the remodelling in bone tissue, the persistence of oral micro-trauma, and the oral mucosal or odontogenic inflammation and infection [4]. MRONJ significantly affects the patient's quality of life (QoL), suffering from chronic pain, bone infections, tooth loss and compromised oral function. Thus, it is crucial increasing the knowledge of its pathogenesis and management, leading the decision-making process of specialists. This study retrospectively describes the clinicalpathological features of MRONJ cases, with a minimal 2-years follow-up, related to oncological and osteometabolic patients treated with Denosumab at the "Ospedali Riuniti", Ancona General Hospital.

Materials and Methods: The study included all MRONJ cases of oncological and osteometabolic patients administered with Denosumab with or without others ONJ-related drugs, diagnosed from January 2014 to December 2018 in the Department of Special and Surgical Stomatology, "Ospedali Riuniti”, Ancona General Hospital. The following clinicalpathological data were collected from all patients: age, gender, date of diagnosis, type of solid primary cancer, or myeloma, or history of osteoporosis, MRONJ site, drug-related, systemic, and local risk factors, clinical and radiographic examinations, MRONJ stage, surgical management, follow-up period, and clinical outcome. Data were retrieved and catalogued from clinical records of the Department of Special and Surgical Stomatology, "Ospedali Riuniti”, Ancona General Hospital, Italy, by a single operator, to ensure their uniformity. All patients were administered Denosumab, according to the following protocols: Xgeva®, fl. $120 \mathrm{mg}$, subcutaneous injections/28 days for cancer patients or Prolia $\AA^{\circledR}$, fl. $60 \mathrm{mg}$, subcutaneous injections / 6 months for osteometabolic ones. Clinical and radiological follow-up were conducted at $10^{\text {th }}$ and $30^{\text {th }}$ day and every 2 months from the initial surgical treatment, for 2 -years at least, to determinate patient's clinical outcome.

Results: Between January 2014 and December 2018, 20 cases of Denosumab-related ONJ were diagnosed. Especially, half of cases were collected during 2017 and two thirds of them were diagnosed between 2017 and 2018 . The age-range varied between 43 and 86 years, with a mean age at diagnosis of $65.5 \pm 11.7$ years, and a slightly late incidence in male gender (69 \pm 11.6 years). Female was the most affected gender (14/20 cases), reporting a mean age at diagnosis equal to $63.9 \pm 11.9$ years. The $90 \%$ of patients (18/20) were oncological cases, undergoing to antiresorptive therapy for bone metastases of breast cancer (11/20), prostate cancer (4/20), renal carcinoma (1/20) and multiple myeloma (2/20). The mean Denosumab treatment duration was equal to $24 \pm 24.8$ months with an average cumulative dose of $2.7 \pm 3.1 \mathrm{~g}$. Before Denosumab, six patients received BFs, mainly zoledronic acid intravenous, with a mean duration of the entire treatment equal to $71 \pm 62.7$ months. Finally, two patients assumed Denosumab together with biological agents (Bevacizumab, Axitinib). At pharmacological history, 6 patients received concurrent chemotherapy and 2 patients were treated by systemic corticosteroids. About the 50\% (9/20) of patients were smokers; third of them were classified as "heavy smokers" ( $\geq 20$ cigarettes/day). At medical history, the arterial hypertension and the hypovitaminosis D represented the 
most common systemic comorbidities. Moreover, sporadic cases of rheumatoid arthritis and diabetes mellitus were recorded. On intraoral examination, a quarter of patients were affected by endodontic and/or periodontal lesions and half of cases showed poor oral hygiene. The mandible was the most affected site (13/20), especially the posterior mandibular region (11/13). Endodontic and/or periodontal abscess and pain were the most complained clinical signs and symptoms (8/20). Most of cases were diagnosed as stage $2(11 / 20)$ and stage $1(8 / 20)$, whereas only one case of stage 3 MRONJ was reported. Orthopantomography was performed to all cases, whereas a quarter of patients also executed second-level imaging (computer tomography). Surgical treatment represented the elective therapy for all patients. Especially, 12 cases underwent to surgical resection, and 8 patients were treated by surgical debridement with or without platelet rich fibrin (PRF; 3/8). During the follow-up (range: 27-53 months), 3 disease relapses were diagnosed after a disease-free survival time equal of $33.3 \pm 6.5$ months.

Conclusions: MRONJ is a common adverse event in oncological patients, and it should require continuous attention and awareness by prescribers, oral and maxillofacial specialists, dental practitioners, as well as health policy stakeholders. Increasing the knowledge of its pathogenesis and management is crucial to improvement the patients QoL and the decision-making process of equipe involved in its primary and secondary prevention. Reduced bone remodelling and infection or inflammation are considered central to its pathogenesis. Indeed, increasing evidence have shown that immunity dysfunction induced by antiresorptive drugs modify the mucosal and the osteoimmune homeostasis, impairing the resistance to infection and the injury repair ${ }^{[4]}$. The real incidence and prevalence of MRONJ in general population are unknown and highly variable, as epidemiological data is scarce. The sources of data derive from randomized trials, observational, retrospective, systematic population studies, case series and surveys of specialists or specialistic units. Despite the efficacy of prevention measures, the reduction of long-term BFs prescription, and the introduction of Denosumab, literature data showed a substantially unchanged number of new cases/year. The ONJ risk in oncohaematological patients undergoing to a long-term intravenous BPs ranges between 1\% and 10\%, about 2-years later the treatment beginning, whereas, its prevalence in non-cancer patients is estimated overall between $0.02 \%$ and $1 \%$. MRONJ incidence in oncological patients could be influenced by the malignancy type and severity, by the assumption of other systemic drugs, and by the higher doses used for the bone disease management, compared to those administer in osteometabolic patients. Denosumab-related ONJ prevalence in oncological patients is estimated at 1-2\%; while in osteometabolics no significant case series have been published to evaluate its epidemiology ${ }^{[3]}$. Our data showed a higher prevalence for 2017-2018 years compared to the previous triennium. It could be attribute to the introduction of Denosumab for solid cancer Italian population in $2013^{[5]}$ and to the onset of Denosumab-related ONJ approximately after 2-3 years of treatment ${ }^{[6]}$. Furthermore, the two-year period 2017-2018 includes two thirds of the cases undergoing to "drug switch" from BFs to Denosumab, which received a higher cumulative dose. Finally, the increasing of knowledge regarding MRONJ may have improved the diagnostic sensitivity of specialists ${ }^{[5]}$. Our results show a higher prevalence of MRONJ in female gender ${ }^{[5]}$, in breast cancer patients ${ }^{[5]}{ }^{[7]}$ and in subject administered with concurrent chemotherapy, as reported in literature ${ }^{[3][5]}$. Clinically, the mandible represents the most affected site, pain was the most symptom complained, and stage 2 was the most common diagnosed, according to literature ${ }^{[2]}\left[{ }^{[5]}\right.$. To the Italian recommendations, the diagnostic therapeutic - assistance path should evaluate all the pharmacological therapies and perform a thorough physical examination and medical history, together with targeted radiologic examinations. It is no longer necessary the presence of 
exposed necrotic bone or bone probing and pain may not always be present, especially in the early stages. The bone biopsy is recommended only in the suspicious of primitive or metastatic oral cancer. Finally, some cases of MRONJ can arise from the presence of dental-periodontal diseases or spontaneously, without any relation to invasive dental procedures ${ }^{[1]}[3]$. Accurate studies are not yet available in literature, due to the heterogeneity of the exposed population, the variability of clinical data collection, the changing and the innovation of the therapeutic protocol and the discrepancy between sources of data. Therefore, is fundamental improvement the scientific research, reporting any cases of new drugs, to clarify by evidence-based medicine all the controversial aspects of MRONJ.

\section{References}

1. a, bGiuseppina Campisi, Rodolfo Mauceri, Francesco Bertoldo, Giordana Bettini, et al. (2020). Medication-Related Osteonecrosis of Jaws (MRONJ) Prevention and Diagnosis: Italian Consensus Update 2020. IJERPH, vol. 17 (16), 5998. doi:10.3390/ijerph17165998.

2. a, bOurania Nicolatou-Galitis, for the MASCC Bone Study Group, Maria Kouri, Erofili Papadopoulou, et al. (2018). Osteonecrosis of the jaw related to non-antiresorptive medications: a systematic review. Support Care Cancer, vol. 27 (2), 383-394. doi:10.1007/s00520-018-4501-x.

3. a, b, c, dCampisi G., Bedogni A., Fusco V. (2020). Raccomandazioni clinico-terapeutiche sull'osteonecrosi delle ossa mascellari (ONJ) farmaco-relata e sua prevenzione. Palermo University press.

4. ${ }^{a}$, b Weidong Zhang, Ling Gao, Wenhao Ren, Shaoming Li, et al. (2021). The Role of the Immune Response in the Development of Medication-Related Osteonecrosis of the Jaw. Front. Immunol., vol. 12 . doi:10.3389/fimmu.2021.606043.

5. a, b, c, d, e, f V. Fusco, M. Cabras, F. Erovigni, A. Dell'Acqua, et al. (2020). A multicenter observational study on Medication-Related Osteonecrosis of the Jaw (MRONJ) in advanced cancer and myeloma patients of a cancer network in North-Western Italy. Med Oral. doi:10.4317/medoral.24318.

6. 'Maura Rossi, Alessio Gambino, Marco Cabras, Manuela Alessio, et al. (2021). Persistence, compliance. discontinuation rates and switch rates in denosumab and bisphosphonate treatment of bone metastases in cancer patients: reasons of switch, osteonecrosis of the jaw (ONJ) and other critical points. Support Care Cancer. doi:10.1007/s00520-021-06100-4.

7. 'Charumathi Raghu Subramanian, Swapna Talluri, Sanjana Mullangi, Manidhar R Lekkala, et al. (2021). Review of Bone Modifying Agents in Metastatic Breast Cancer. doi:10.7759/cureus.13332. 